\title{
EVALUACIÓN DEL APROVECHAMIENTO DE BONELLIA SPRUCEI (Mez) COMO PRODUCTO FORESTAL NO MADERABLE EN DOS LOCALIDADES DE LA COMUNIDAD JULCUY
}

\author{
AUTORES: Alfredo Jiménez González ${ }^{1}$ \\ Cecibel Margarita Conforme Quimis ${ }^{2}$ \\ Paola Yadira Moreira Aguayo ${ }^{3}$ \\ Lucy Maritza García Lucas ${ }^{4}$
}

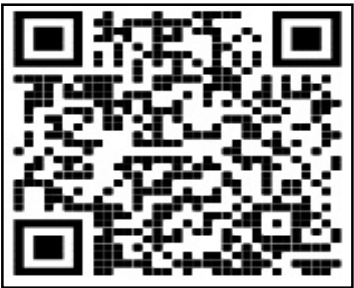

DIRECCIÓN PARA CORRESPONDENCIA: alfredo.jimenez@unesum.edu.ec

Fecha de recepción: 28/07/2020

Fecha de aceptación: 28/11/2020

\section{RESUMEN}

Una evaluación del aprovechamiento de Bonellia sprucei (Mez) como producto forestal no maderable se realizó en las localidades de Las Peñas y Soledad de la localidad de Julcuy, para conocer sobre el aprovechamiento y potencialidades de uso de los Productos Forestales No Maderables derivados de esta especie. Se realizaron recorridos de campo, entrevistas y charlas, así el objetivo del trabajo consistió en evaluar el aprovechamiento de Bonellia sprucei (Mez), en las localidades antes mencionadas, a través del método empírico de encuestas y la realización de un muestreo aleatorio estratificado; para el muestreo se establecieron transectos de $20 \mathrm{~m}$ x $50 \mathrm{~m}$. Mediante un inventario florístico en el bosque seco tropical de aquellas localidades, se comprobó el grado de asociación con otras especies. Así mismo se determinó su abundancia, los porcentajes, cantidad de uso y sus derivados. Los resultados revelan la composición del ecosistema, y demuestran que estos productos no se explotan al máximo. La distancia de los árboles a las viviendas es una variable que incide en el precio final del producto.

PALABRAS CLAVE: etnobotánica; comercialización; vegetal: frutos.

\footnotetext{
${ }^{1}$ Dr. En Ciencias Forestales, Ingeniero Agrónomo. Profesor Titular Principal 1. Carrera de Ingeniería Forestal, Universidad Estatal del Sur de Manabí. Km 1 1 1/2 Vía Novoa s/n Campus Los Ángeles, Jipijapa, Manabí, Ecuador. CP. 130 650, e-mail: alfredo.jimenez@unesum.edu.ec , teléf.: 593+996163589. https://orcid.org/0000-0002-1768-5566 ${ }^{2}$ Ing. Forestal, Carrera de Ingeniería Forestal, Universidad Estatal del Sur de Manabí. Universidad Estatal del Sur de Manabí. Km $1 \frac{1}{2}$ Vía Novoa s/n Campus Los Ángeles, Jipijapa, Manabí, Ecuador, e-mail: maggytta31@hotmail.com. https://orcid.org/0000-0003-0998-3691

${ }^{3}$ Licenciada En Ciencias De La Educación Especialidad Inglés, Magister En Enseñanza Del Idioma Inglés. Directora del Centro de Idiomas de la Universidad Estatal del Sur de Manabí. Km 1 1⁄2 Vía Novoa s/n Campus Los Ángeles, Jipijapa, Manabí, Ecuador, e-mail: paola.moreira@unesum.edu.ec. https://orcid.org/0000-0001-6764-3156

${ }^{4}$ Ing. Forestal, Magister en Manejo y Aprovechamiento Forestal, Carrera de Ingeniería Forestal, Universidad Estatal del Sur de Manabí. Universidad Estatal del Sur de Manabí. Km 1 1⁄2 Vía Novoa s/n Campus Los Ángeles, Jipijapa, Manabí, Ecuador, e-mail: negrito_alan@live.com. https://orcid.org/0000-0002-0267-6165
} 
Alfredo J. González, Cecibel M.Conforme Quimis, Paola Y. Moreira Aguayo, Lucy M. García Lucas

\title{
EVALUATION OF THE USE OF BONELLIA SPRUCEI (Mez) AS A NON- TIMBERABLE FOREST PRODUCT IN TWO VILLAGES OF THE JULCUY COMMUNITY
}

\begin{abstract}
An evaluation of the use of Bonellia sprucei Mez as a non-wood forest product was carried out in the towns of Las Peñas and Soledad in the town of Julcuy, to learn about the use and potential of the use of Non-Wood Forest Products derived from this species. Field trips, interviews and talks were carried out, thus the objective of the work was to evaluate the use of Bonellia sprucei (Mez), in the aforementioned localities, through the empirical method of surveys and the performance of a stratified random sampling; for the sampling, $20 \mathrm{~m} \times 50 \mathrm{~m}$ transects were established. Through a floristic inventory in the tropical dry forest of those localities, the degree of association with other species was verified, as well as its abundance, percentages, amount of use and its derivatives were determined. The results reveal the composition of the ecosystem, and show that these products are not fully exploited. The distance from the trees to the houses is a variable that affects the final price of the product.
\end{abstract}

KEYWORDS: Ethnobotany; commercialization; vegetable; fruits.

\section{INTRODUCCIÓN}

La FAO (2018) en la Agenda 2030 para el Desarrollo Sostenible señalan que: Un compromiso asumido por los países para hacer frente a los complejos desafíos que se plantean, desde poner fin a la pobreza y el hambre y responder al cambio climático hasta crear comunidades resilientes, lograr un crecimiento inclusivo y gestionar los recursos naturales de la Tierra de forma sostenible (p. 12).

En el informe de la FAO en 2018 menciona que, los bosques representan una fuente de alimentos, medicinas y combustible para más de mil millones de personas. Además de ayudar a responder al cambio climático y proteger los suelos y el agua, albergan más de tres cuartas partes de la biodiversidad terrestre mundial, proporcionan numerosos productos y servicios que contribuyen al desarrollo socioeconómico y son particularmente importantes para cientos de millones de moradores de las zonas rurales, entre los que se cuentan muchas de las personas más pobres del mundo.

Por lo general, cuando se habla de bosques y selvas se piensa solo en árboles y en la madera que de ellos se extrae. Esta visión, es la herencia de una forma errónea de ver la naturaleza y de los viejos modelos "extractivos" de explotación de los recursos naturales, deja de lado a la mayoría de las especies de la flora y la fauna que, junto con los árboles, constituyen lo que se conoce como ecosistemas forestales, en los cuales existe un sinnúmero de plantas y animales que, asociados con los árboles de valor comercial reconocido o "maderables" aportan numerosos bienes y servicios dentro de los que se destacan los productos alimenticios (Lopez, 2008).

De acuerdo con estudios realizados por la Organización de las Naciones Unidas para la Alimentación y la Agricultura (FAO), en todo el mundo existe dependencia de los Productos Forestales No Madereros (PFNM), para su subsistencia y para la obtención de ingresos. La 
población rural, en particular, depende de estos productos como fuentes de subsistencia (FAO, 2014a).

A nivel local, los PFNM también se utilizan como materia prima para la elaboración industrial a gran escala. Recientemente los PFNM han suscitado un interés considerable por su importancia cada vez más reconocida y la consecución de objetivos ambientales como la conservación de la diversidad biológica (FAO, 2014a).

Nativa de la costa ecuatoriana, Bonellia sprucei es una de especie del Bosque Seco Pluviestacional, distribuida geográficamente, en las provincias de El Oro, Guayas y Manabí (Hassler, 2019). La presente investigación aborda la problemática relacionada con la sustentabilidad en el aprovechamiento de Bonellia sprucei como PFNM, en las localidades de Las Peñas y Soledad de la parroquia Julcuy, por lo que se presenta el objetivo de evaluar el aprovechamiento de Bonellia sprucei como producto forestal no maderable en las localidades de Las Peñas y Soledad de la parroquia Julcuy del cantón Jipijapa. .

\section{Ubicación geográfica}

La localidad las Peñas y la localidad Soledad pertenecientes a la parroquia Julcuy del cantón Jipijapa.

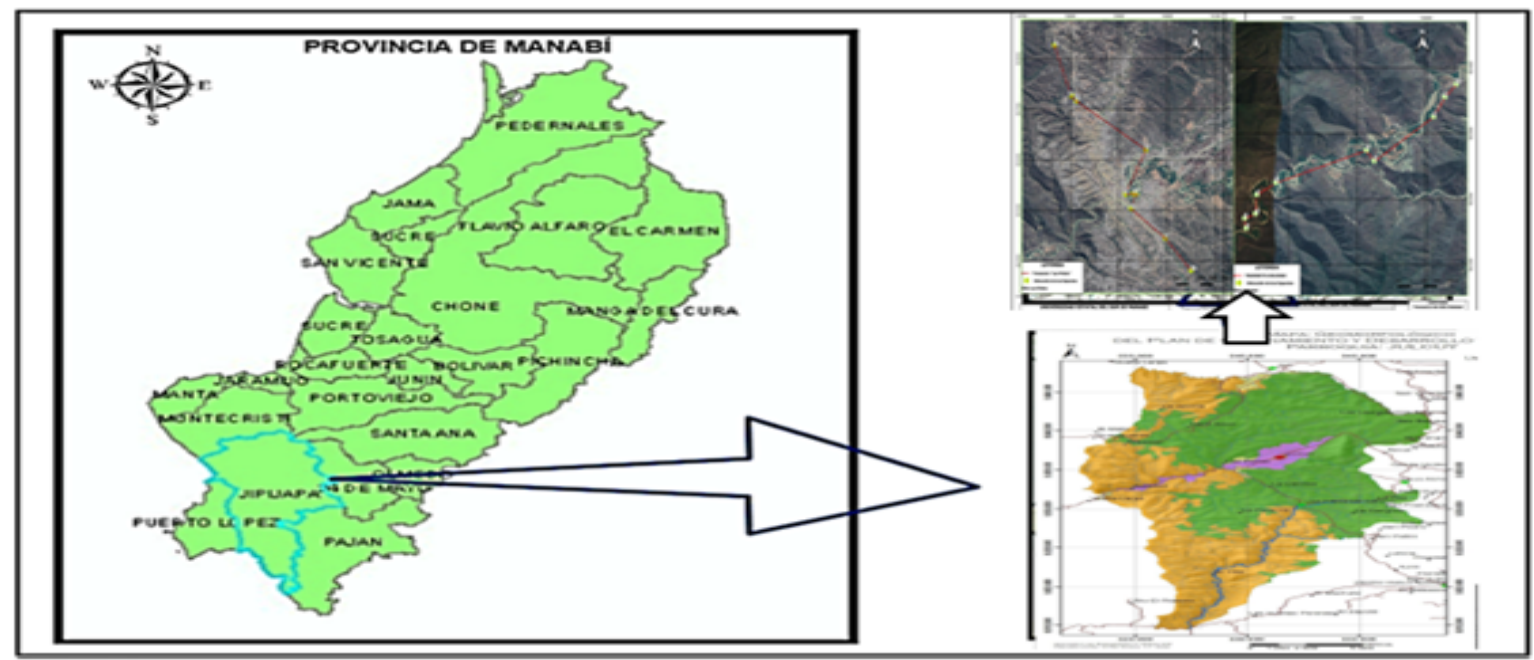

Figura 1. Localidades de estudio en la parroquia Julcuy, del cantón Jipijapa.

El clima de Julcuy es un clima estepa local, durante el año hay poca lluvia. De acuerdo con Köppen y Geiger el clima se clasifica como BSh (Seco Estepario cálido). La temperatura media anual en Julcuy se encuentra a $23,8^{\circ} \mathrm{C}$. La precipitación es de $511 \mathrm{~mm}$ al año. La precipitación es la más baja en agosto, con un promedio de $4 \mathrm{~mm}$. La mayor parte de la precipitación aquí cae en marzo, promediando $126 \mathrm{~mm}$. A una temperatura media de $25,2^{\circ} \mathrm{C}$, marzo es el mes más caluroso del año y julio es el mes más frío, con temperaturas promediando $22,6^{\circ} \mathrm{C}$. Entre los meses más secos y más húmedos, la diferencia en las precipitaciones es $122 \mathrm{~mm}$. A lo largo del año, las temperaturas varían en $26^{\circ} \mathrm{C}$ (CLIMATE-DATA.ORG, 2019). En la región predomina el Bosque seco pluvio-estacional (Aguirre, 2012a). 


\section{Metodología}

Se realizaron recorridos de campo para constatar in situ las potencialidades y usos de la especie Bonellia sprucei en las localidades de Las Peñas, y Soledad, lo cual se logró mediante el dialogo con las personas de las localidades.

Se realizaron visitas a dichas localidades para solicitar permisos para investigación, además se participó en los talleres y charlas realizados en el marco del proyecto "Componentes de la diversidad biológica empleados por las familias manabitas en la medicina natural y tradicional", de la carrera de Ingeniería forestal, aprobado por RESOLUCIÓN N.07-16-2019 del Órgano Colegiado Académico Superior de la Universidad Estatal del Sur de Manabí, en sesión extraordinaria celebrada el 06 de mayo del 2019. Programa al que pertenece - Ecoturístico Forestal.

Se utilizó el método de encuestas con el apoyo de las descripciones de Jiménez et al., (2010); Aguirre, (2012b); Jiménez et al., (2017); Jiménez et al., (2019). La muestra para la encuesta etnobiológica fue tomada a personas que habitan dentro de las localidades de Las Peñas, y Soledad, 32 y 45, respectivamente, ambos sitios, ubicados en la parroquia Julcuy. Una vez que conocida la población vinculada con las actividades en el bosque y sus componentes biológicos (en Julcuy), se calculó el número de personas a encuestar, para lo cual se utilizó la fórmula planteada por Morales (2012). Resultando encuestar a 32 personas en la localidad de las peñas y 45 personas en Soledad. Según estos autores, cuando se conoce el tamaño de la población, la muestra necesaria es más pequeña y su tamaño se determina mediante la ecuación [1]:

$$
n=\frac{N}{\frac{1+e^{2(N-1)}}{z^{2 p q}}}
$$

Dónde:

$\mathrm{n}=$ tamaño de la muestra que deseamos conocer

$\mathrm{N}=$ tamaño conocido de la población

e: error que se prevé cometer. Y como no se requiere un error mayor del $3 \%$, se tiene que e $=$ 0,03 .

$\mathrm{z}=1,96$ para un nivel de confianza del $95 \%, \alpha=0,05$

pq: varianza de la población $=0,25$

De acuerdo con Morales (2012), como la varianza de la población se desconoce, se coloca la varianza mayor posible porque a mayor varianza hará falta una muestra mayor. La varianza en los ítems dicotómicos (dos respuestas que se excluyen mutuamente) es igual a pq y la varianza mayor (la mayor diversidad de respuestas) se da cuando $\mathrm{p}=\mathrm{q}=0,50$ (la mitad de los sujetos responde sí y la otra mitad responde no) por lo que en esta fórmula [1] pq es siempre igual a $(0,50)(0,50)=0,25$ (es una constante) . 
e: error que se prevé cometer. Y como no se requiere un error mayor del $3 \%$, se tiene que e $=$ 0,03 .

El tamaño de la muestra se realizó tomando el $8 \%$ de la población total de la parroquia. La encuesta se aplicó con el fin de conocer el uso y aprovechamiento de Bonellia sprucei en ambas localidades. Teniendo en cuenta las características de esta población, se hicieron preguntas sencillas de SI y NO con un grupo de variables.

\section{Riqueza de especies}

La curva de riqueza de especies, se confeccionó para determinar si el esfuerzo de muestreo fue suficiente para representar debidamente la comunidad estudiada. Para esto se utilizó el software BioDiversity Pro Versión 2.0 para Windows (1997).

Se realizaron gráficos de rango-abundancia para las especies inventariadas en cada localidad. Las curvas se realizaron a escala logarítmica, por lo que cada valor de abundancia será transformado a $L_{2}$ de cada Pi, Feinsinger (2004) y Jimenez (2012), dado por la ecuación [2]:

$$
P i=n i / N[2]
$$

Donde:

nies el número de individuos de la especie $\mathrm{i}$

$\mathrm{N}$ es el número total de individuos

Pi es la proporción de los individuos en una comunidad o una muestra que pertenece a la especie i.

Las especies de cada muestra están graficadas de mayor a menor abundancia (del Pi más alto al más bajo), dentro de esa muestra. Las curvas fueron elaboradas mediante elGraphPadPrism versión 8.0.0 para Windows, GraphPad Software.

\section{Tamaño de los transectos}

Para realizar el muestreo se tuvo en cuenta los criterios de (Aguirre 2012a). Este autor planteo que para evaluar productos arbóreos, como es el caso de Bonellia sprucei, se pueden utilizar parcelas o transectos, estos últimos de $50 \mathrm{~m}$ x $20 \mathrm{~m}\left(1000 \mathrm{~m}^{2}\right)$. Así mismo que el número de unidades de muestreo debe ser al menos cinco, distribuidas a una distancia entre $100 \mathrm{~m}$ a $200 \mathrm{~m}$, para lo que se debe seguir un gradiente altitudinal, además de considerar la información previa relacionada con la existencia de los PFNM en la zona. En este estudio se muestrearon 20 transectos, 10 en cada localidad.

\section{Descripción de los instrumentos}

En la elaboración de este instrumento de muestreo se tuvo en cuenta los criterios de la FAO (2000), relacionada con la evaluación y el monitoreo de toda la variedad de productos forestales que dan origen a los PFNM en un país determinado. La selección del método de encuestas se 
basó en los planteamientos de Wong, Kirsti \& Nell (2001), citados por Jiménez et al., (2010) y Jiménez et al., (2017), al referirse a las técnicas de ciencias sociales como uno de los métodos más efectivos para la obtención de un inventario de los PFNM.

La encuesta sobre el uso de los Productos Forestales no Maderables (PFNM) derivados de la especie Bonellia sprucei en dos localidades, Las Peñas y Soledad de la parroquia Julcuy; consta de 13 preguntas y se constituyó para indagar en la muestra algunos aspectos etnobiológicos y personales.

Los aspectos personales censados en la encuesta fueron:

- La edad

- El sexo

- Nivel de educación

Para describir la edad de los pobladores encuestados en las localidades de Las Peñas y Soledad se tomaron como referencia cuatro rangos de edades, de 10 años cada uno.

Pregunta 1.- ¿Qué tiempo hace que usted reside en esta localidad?

Pregunta 2.- ¿Conoce usted el árbol de barbasco?

Pregunta 3.- ¿Existe el barbasco en su localidad?

Pregunta 4 - ¿Aprovecha usted el barbasco con alguna finalidad? En caso positivo, ¿para qué la aprovecha?

Pregunta 5.- ¿Qué partes de la planta aprovecha?

Pregunta 6.- ¿Cómo utiliza la parte de la planta que ha señalado?

Pregunta 7.- Ambiente donde crece la planta (hábitat)

Pregunta 8.- ¿Con qué frecuencia se dirige al bosque con la finalidad de aprovechar el barbasco como PFNM?

Pregunta 9.- ¿En una escala del 1 al 5, siendo el 5 el máximo, en qué magnitud aprovecha el barbasco como PFNM? Escala: 1 - muy bajo, 2- bajo; 3- Medianamente alto; 4- alto; 5- muy alto.

Pregunta 10.- ¿En una escala del 1 al 5, siendo el 5 el máximo, cuál es su percepción de la abundancia del barbasco en su localidad? Escala: 1 - muy bajo, 2- bajo; 3- Medianamente alto; 4alto; 5- muy alto.

Pregunta 11.- Distancia en $\mathrm{km}$ desde la vivienda hasta el bosque donde colectan el barbasco como PFNM.

Pregunta 12.- Objeto de la cosecha del producto.

Pregunta 13.- Época de recolección del producto.

El porcentaje de usos de las especies se calculó según los criterios de Molares et al. (2009); Aguirre (2012a) y Jiménez et al., (2017), mediante la pregunta uno de la encuesta, relacionada con los PFNM que utiliza del bosque, a través de la ecuación [3]: 


$$
\% \text { deusodeunaespecie }=\frac{f n}{N} 100[3]
$$

Dónde:

fn: Frecuencia absoluta de la especie

$\mathrm{N}$ : Número total de citaciones por parte de los encuestados.

La pregunta cinco se ejecutó para determinar qué partes de las plantas utilizan para diversos fines, en este caso se tiene en cuenta solo el fruto de la especie.

La forma de usos de la parte de la planta que aprovecha, construcción, alimento, artesanías, ornamental y otros, se constituyó en la pregunta seis.

La séptima pregunta se concretó en qué ambiente crece el árbol de barbasco, por ejemplo: bosque, matorral, áreas abiertas, riveras de quebradas/ríos.

La frecuencia con que se dirigen al bosque los habitantes de las localidades de Las Peñas y Soledad fue planteada a través de tres rangos de tiempo, a saber: $1-3$ meses muy frecuente, $4-$ 5 meses medianamente frecuente, $6-7$ meses poco frecuente. Esta descripción corresponde a la octava pregunta.

La pregunta nueve está relacionada con la cantidad de barbasco que aprovechan. Así mismo, la décima pregunta indagó sobre su percepción acerca de la abundancia del barbasco en su localidad.

Para describir la distancia que existe entre las viviendas de los pobladores de las localidades de Las Peñas y Soledad hacia el sitio donde la aprovechan el barbasco (pregunta 11), se consideraron cinco rangos de distancia: $0-5 \mathrm{~km} ; 6-10 \mathrm{~km} ; 11-15 \mathrm{~km} ; 16-20 \mathrm{~km}$ y por último, más de $21 \mathrm{~km}$.

Para indagar sobre el objeto de la cosecha del producto, en este caso: venta, consumo y ventaconsumo se preparó la pregunta 12 .

La pregunta 13 guarda una estrecha relación con la productividad y capacidad de recuperación del ecosistema, para lo cual se indagó acera de: la época de recolección del producto, en este caso: temporada lluviosa, temporada seca y todo el año. Se considera muy importante conocer o describir la época del año que escogen los pobladores de las localidades de Las Peñas y Soledad, debido a la escasez de agua, donde las plantas quedan ostentadas, a las presiones naturales del clima y a las presiones de origen antrópico que favorecen a la degradación de la tierra y a la pérdida de la diversidad biológica.

\section{RESULTADOS}

Porcentajes y cantidades de usos de los PFNM derivados de Bonellia sprucei en las localidades de Las Peñas y Soledad de la Parroquia Julcuy

En la (Tabla 1) se presentan los porcentajes de usos de los PFNM, en cada comunidad estudiada, calculado mediante el número de citaciones y la frecuencia de uso por categoría. 
Tabla 1 . Porcentajes y cantidades de uso de los PFNM derivados de Bonellia sprucei en las localidades de Las Peñas y Soledad de la parroquia Julcuy

\begin{tabular}{lllc}
\hline Comunidad & & Venta - comercializan & N \\
\hline \multirow{3}{*}{ Las Peñas } & $\begin{array}{l}\text { Citaciones/frecuencia de } \\
\text { uso por categoría }\end{array}$ & 32 & 80 \\
\cline { 2 - 4 } & $\begin{array}{l}\mathrm{fn}=\% \text { de uso de las } \\
\text { especies de PFNM }\end{array}$ & 40 & 100 \\
\hline \multirow{3}{*}{ Soledad } & $\begin{array}{l}\text { Citaciones/frecuencia de } \\
\text { uso por categoría }\end{array}$ & 45 & 100 \\
\cline { 2 - 4 } & $\begin{array}{l}\mathrm{fn}=\% \text { de uso de las } \\
\text { especies de PFNM }\end{array}$ & 45 & 100 \\
\hline fn: Frecuencia absoluta de la especie; N: Número total de citaciones por parte de los encuestados.
\end{tabular}

En la localidad de Las Peñas solo se dedican a vender los frutos de esta especie la venden por canecas (tacho de $20 \mathrm{~L}$ ), los que se dedican a recolectar y a vender reciben 3,00 USD (dólares americanos) recibiendo un total de 9,00 USD (dólares americanos) por un saco lleno.

De acuerdo con los resultados de la (Tabla 1), para la localidad de Las Peñas, se muestra entre los principales usos y aprovechamiento, la venta y comercialización, en tanto que, en Soledad el porcentaje no varía ya que también se dedican a la misma actividad.

En la (Tabla 2) se presentan los resultados de la pregunta relacionada con el tiempo que residen en su localidad.

Tabla 2. Cantidad de personas encuestadas/ años de residencia en las localidades de Las Peñas y Soledad

\begin{tabular}{|l|l|l|l|l|}
\hline & \multicolumn{2}{|c|}{ Las Peñas } & \multicolumn{2}{c|}{ Soledad } \\
\hline Tiempo/Años & Cantidad & $\mathbf{\%}$ & Cantidad & $\%$ \\
\hline $0-10$ años & 6 & 19 & 7 & 16 \\
\hline $11-20$ años & 4 & 13 & 10 & 22 \\
\hline $21-30$ años & 3 & 9 & 8 & 18 \\
\hline $31-40$ años & 7 & 22 & 12 & 27 \\
\hline $41-$ años y más & 12 & 38 & 8 & 18 \\
\hline Total & 32 & $\mathbf{1 0 0}$ & $\mathbf{4 5}$ & $\mathbf{1 0 0}$ \\
\hline
\end{tabular}

Como se observa en la Tabla anterior en la localidad de las Peñas el mayor número de personas que residen están en el rango de 41- años y más, a diferencia con la localidad de Soledad que la mayor parte de los encuestados tienen entre los 31 y los 40 años de permanencia en el lugar, más del $20 \%$ de los encuestados.

En la (Tabla 3) se presentan los resultados sobre el conocimiento de la especie objeto de estudio, Bonellia sprucei.

Tabla 3. Conocimiento de la especie Bonellia sprucei por los pobladores de las localidades de Las Peñas y Soledad 


\begin{tabular}{|l|c|c|c|c|}
\hline \multirow{2}{*}{ Conocimiento de la especie } & \multicolumn{2}{|c|}{ Las Peñas } & \multicolumn{2}{c|}{ Soledad } \\
\cline { 2 - 5 } & Cantidad & $\mathbf{\%}$ & Cantidad & $\mathbf{\%}$ \\
\hline $\mathrm{Si}$ & 32 & 100 & 45 & 100 \\
\hline No & 0 & & 0 & 0 \\
\hline Total & $\mathbf{3 2}$ & $\mathbf{1 0 0}$ & $\mathbf{4 5}$ & $\mathbf{1 0 0}$ \\
\hline
\end{tabular}

Como se muestra la tabla de la segunda pregunta la totalidad de los encuestados tanto en Las Peñas y Soledad sus respuestas fueron afirmativas.

En la (Tabla 4) se presentan los resultados de la pregunta relacionada con la existencia de la Bonellia sprucei en su localidad, lo que corresponde a la pregunta 3.

Tabla 4. Presencia de la especie Bonellia spruceien sus localidades de residencia

\begin{tabular}{|l|l|l|l|l|}
\hline \multirow{2}{*}{ Existencia de la especie } & \multicolumn{2}{|c|}{ Las Peñas } & \multicolumn{2}{c|}{ Soledad } \\
\cline { 2 - 5 } & Cantidad & $\mathbf{\%}$ & Cantidad & $\%$ \\
\hline $\mathrm{Si}$ & 32 & 100 & 45 & 100 \\
\hline No & 0 & & 0 & \\
\hline Total & $\mathbf{3 2}$ & $\mathbf{1 0 0}$ & $\mathbf{4 5}$ & $\mathbf{1 0 0}$ \\
\hline
\end{tabular}

Luego de indagar sobre el conocimiento relacionado con la existencia de la especie objeto de estudio, se comprobó que todas las personas encuestadas corroboraron de manera afirmativa la presencia del árbol de Bonellia sprucei en ambas localidades.

En la (Tabla 5) se presentan los resultados del aprovechamiento o no de Bonellia sprucei, así como con qué finalidad.

Tabla 5. Usos del aprovechamiento de Bonellia sprucei que hacen los pobladores de las comunidades de Las Peñas y Soledad.

\begin{tabular}{|l|l|l|l|l|}
\hline \multirow{2}{*}{$\begin{array}{l}\text { Usos fos finalidad de } \\
\text { aprovechamiento }\end{array}$} & \multicolumn{2}{|c|}{ Las Peñas } & \multicolumn{2}{c|}{ Soledad } \\
\cline { 2 - 5 } & Cantidad & $\mathbf{\%}$ & Cantidad & $\mathbf{\%}$ \\
\hline Alimentos y Bebidas & 0 & 0 & 0 & 0 \\
\hline $\begin{array}{l}\text { Tóxicos: } \\
\text { Pescar/lavar/insecticida }\end{array}$ & 0 & 0 & 0 & 0 \\
\hline Otros (Venta) & 32 & 100 & 45 & 100 \\
\hline Total & $\mathbf{3 2}$ & $\mathbf{1 0 0}$ & $\mathbf{4 5}$ & $\mathbf{1 0 0}$ \\
\hline
\end{tabular}

En las localidades de Las Peñas y Soledad el $100 \%$ de los encuestados solo utilizan el fruto de la especie y solo la venden a comerciantes intermediarios.

En la (Tabla 6) se muestran los resultados de la pregunta 5, relacionada con las partes de la planta que aprovechan. 
Tabla 6. Partes de Bonellia sprucei que aprovechan los pobladores de las localidades de Las

\begin{tabular}{|l|l|l|l|l|}
\hline \multirow{4}{*}{ Partes de la planta } & \multicolumn{2}{c|}{ Las Peñas } & \multicolumn{2}{c|}{ Soledad } \\
\cline { 2 - 5 } & Cantidad & $\mathbf{\%}$ & Cantidad & $\%$ \\
\hline Raíz & 0 & 0 & 0 & 0 \\
\hline Tallo & 0 & 0 & 0 & 0 \\
\hline Hojas & 0 & 0 & 0 & 0 \\
\hline Flores & 0 & 0 & 0 & 0 \\
\hline Ramas & 0 & 0 & 0 & 0 \\
\hline Frutos & 32 & 100 & 45 & 100 \\
\hline Corteza & 0 & 0 & 0 & 0 \\
\hline Total & $\mathbf{3 2}$ & $\mathbf{1 0 0}$ & $\mathbf{4 5}$ & $\mathbf{1 0 0}$ \\
\hline
\end{tabular}

La parte aprovechada del árbol de barbasco en las dos localidades es el fruto y este solo es utilizado para la venta.

En la (Tabla 7), se presentan los resultados de la sexta pregunta de la encuesta, relacionada con la manera cómo utilizan la parte del árbol mencionada en la (Tabla 8).

Tabla 7. Usos que le dan los encuestados en las comunidades de Las Peñas y de Soledad a las partes de la planta de Bonellia sprucei

\begin{tabular}{lllll}
\hline \multirow{2}{*}{ Uso } & Las Peñas & \multicolumn{3}{l}{ Soledad } \\
\cline { 2 - 5 } & Cantidad & $\mathbf{\%}$ & Cantidad & $\mathbf{\%}$ \\
\hline Construcción & 0 & 0 & 0 & 0 \\
Alimento & 0 & 0 & 0 & 0 \\
Artesanía & 0 & 0 & 0 & 0 \\
Ornamental & 0 & 0 & 0 & 0 \\
Comercializar & 32 & 100 & 45 & 100 \\
Otros & 0 & 0 & 0 & 0 \\
\hline Total & $\mathbf{3 2}$ & $\mathbf{1 0 0}$ & $\mathbf{4 5}$ & $\mathbf{1 0 0}$ \\
\hline
\end{tabular}

La parte de Bonellia sprucei que utilizan los habitantes de las dos localidades es comercializado en el centro de acopio el cual está ubicado en la localidad de Las Peñas de la parroquia Julcuy.

En la (Tabla 8) se muestran los resultados obtenidos de la pregunta 7, relacionada con el ambiente dónde crece el árbol.

Tabla 8. Ambiente donde crecen las plantas de Bonellia sprucei en las localidades Las Peñas y de Soledad.

\begin{tabular}{|l|l|l|l|l|}
\hline \multirow{2}{*}{ Ambiente donde crece la planta } & \multicolumn{2}{|c|}{ Las Peñas } & \multicolumn{2}{c|}{ Soledad } \\
\cline { 2 - 5 } & Cantidad & $\%$ & Cantidad & \% \\
\hline Bosque & 14 & 44 & 21 & 47 \\
\hline Matorral & 0 & 0 & 0 & 0 \\
\hline Áreas Abiertas & 11 & 34 & 13 & 29 \\
\hline
\end{tabular}

230 UNESUM-Ciencias. Publicación cuatrimestral. Vol. 4, No. 4 (Septiembre-Diciembre), Año 2020. 


\begin{tabular}{|l|l|l|l|l|}
\hline Riveras de quebradas/hondonadas & 7 & 22 & 11 & 24 \\
\hline TOTAL & $\mathbf{3 2}$ & $\mathbf{1 0 0}$ & $\mathbf{4 5}$ & $\mathbf{1 0 0}$ \\
\hline
\end{tabular}

Según la encuesta realizada en las dos localidades se determinó que el árbol de Bonellia sprucei se lo encuentra con una probabilidad de más del $40 \%$ en el bosque, con una estimación del $30 \%$ en áreas abiertas y finalmente se comprobó que esta especie crece en riveras de quebradas y hondonadas, resultando 22 y 24 puntos porcentuales, respectivamente.

En la (Tabla 9), se puede comprobar la frecuencia de expediciones al bosque con el fin de aprovechar PFNM de Bonellias prucei, que coincide con la octava pregunta de la encuesta.

Tabla 9 . Frecuencia de visitas al bosque con la finalidad de aprovechar Bonellia sprucei como PFNM, en las de localidades Las Peñas y de Soledad.

\begin{tabular}{|l|l|l|l|l|}
\hline \multirow{2}{*}{ Tiempo/Años } & \multicolumn{2}{|c|}{ Las Peñas } & \multicolumn{2}{c|}{ Soledad } \\
\cline { 2 - 5 } & \multicolumn{1}{|c|}{ Cantidad } & $\mathbf{\%}$ & Cantidad & \multicolumn{1}{c|}{} \\
\hline $1-3$ meses & 9 & 28 & 13 & 29 \\
\hline $4-5$ meses & 20 & 63 & 20 & 44 \\
\hline $6-7$ meses & 3 & 9 & 12 & 27 \\
\hline Total & $\mathbf{3 2}$ & $\mathbf{1 0 0}$ & $\mathbf{4 5}$ & $\mathbf{1 0 0}$ \\
\hline
\end{tabular}

1-3 meses (muy frecuente); 4- 5 meses (medianamente frecuente); $6-7$ meses (poco frecuente)

Como se observa en la tabla anterior los pobladores, presentan mayor frecuencias entre cuatro y cinco meses que regresan a recolectar este PFNM, lo cual sucede en ambas localidades, pocos son los que se dirigen al bosque de 1 a 3 meses a recolectar el producto y otros lo hacen de 6 y 7 meses a aprovechar esta especie.

Tabla 10. Resultados de la magnitud de Bonellia sprucei que aprovechan los pobladores de las localidades de Las Peñas y de Soledad

\begin{tabular}{|l|l|l|l|l|}
\hline \multirow{2}{*}{ Escala } & \multicolumn{2}{|c|}{ Las Peñas } & \multicolumn{2}{c|}{ Soledad } \\
\cline { 2 - 5 } & Cantidad & $\mathbf{\%}$ & Cantidad & $\mathbf{\%}$ \\
\hline 1 & 0 & 0 & 0 & 0 \\
\hline 2 & 0 & 0 & 9 & 20 \\
\hline 3 & 7 & 22 & 11 & 24 \\
\hline 4 & 20 & 63 & 21 & 47 \\
\hline 5 & 5 & 16 & 4 & 9 \\
\hline Total & $\mathbf{3 2}$ & $\mathbf{1 0 0}$ & $\mathbf{4 5}$ & $\mathbf{1 0 0}$ \\
\hline
\end{tabular}

1-muy bajo, 2- bajo; 3- Medianamente alto; 4- alto; 5- muy alto

De acuerdo con los resultados presentados en la (Tabla 10), en la localidad de Las Peñas se constató que la mayor cantidad de encuestados indicaron aprovechar medianamente el fruto del árbol de Bonellias prucei, con los mayores porcentajes siendo 3,4 y 5, lo que coincide con un aprovechamiento muy alto; en tanto que en la localidad de Soledad el aprovechamiento es similar 
a la de la localidad anterior ya que ellos de la venta del fruto de Bonellia sprucei llevan el sustento a sus hogares.

Los resultados de la pregunta 10, relacionada con la percepción de la abundancia del barbasco en las localidades se muestran en la (Tabla 11).

Tabla 11. Percepción que tienen los encuestados de la abundancia de Bonellia sprucei en las localidades de Las Peñas y de Soledad

\begin{tabular}{|l|l|l|l|l|}
\hline \multirow{2}{*}{ Escala } & \multicolumn{2}{|c|}{ Las Peñas } & \multicolumn{2}{c|}{ Soledad } \\
\cline { 2 - 5 } & Cantidad & $\mathbf{\%}$ & \multicolumn{1}{c|}{ Cantidad } & \% \\
\hline 1 & 0 & 0 & 0 & 0 \\
\hline 2 & 0 & 0 & 9 & 20 \\
\hline 3 & 8 & 25 & 11 & 24 \\
\hline 4 & 13 & 41 & 21 & 47 \\
\hline 5 & 11 & 34 & 4 & 9 \\
\hline Total & $\mathbf{3 2}$ & $\mathbf{1 0 0}$ & $\mathbf{4 5}$ & $\mathbf{1 0 0}$ \\
\hline
\end{tabular}

1 - muy bajo, 2- bajo; 3- Medianamente alto; 4- alto; 5- muy alto

En la localidad de Las Peñas la percepción de los encuestados presenta porcentajes que oscilaron entre medianamente alto, alto y muy alto lo que coincide con la abundancia encontrada en el muestreo realizado en los transectos de esa localidad, con un total de 93 individuos, según se muestra en la (Tabla 2).

Por otro lado en la localidad de Soledad más del $47 \%$ tiene la percepción de que la abundancia es alta, mientras que el $9 \%$ no perciben esa abundancia, describiéndola como muy alta, así un 20\% de los encuestados tienen la percepción de que la abundancia de Bonellia sprucei es baja.

La movilización, principalmente desde las viviendas hasta los sitios forestales constituye un reto para los pobladores que viven en o del bosque, por lo que se ha encontrado una estrecha relación entre distancia y extracción de PFNM. Los resultados se presentan en la (Tabla 12).

La tabla 12 muestra los resultados de la indagación acerca del conocimiento de la distancia en $\mathrm{km}$ desde su vivienda, hasta el lugar donde colectan el Bonellia sprucei como PFNM, los pobladores de Las Peñas y Soledad.

Tabla 13. Resultados acerca del conocimiento de la distancia en km hasta el lugar donde colectan el Bonellia sprucei

\begin{tabular}{|l|l|l|l|l|}
\hline \multirow{2}{*}{ Distancia en km } & \multicolumn{1}{|c|}{ Las Peñas } & & Soledad & \\
\cline { 2 - 5 } & Cantidad & \multicolumn{1}{c|}{ C } & Cantidad & \multicolumn{1}{c|}{} \\
\hline $0-5$ & 13 & 41 & 22 & 49 \\
\hline $6-10$ & 11 & 34 & 13 & 29 \\
\hline $11-15$ & 8 & 25 & 10 & 22 \\
\hline $16-20$ & 0 & 0 & 0 & 0 \\
\hline
\end{tabular}




\begin{tabular}{|l|l|l|l|l|}
\hline más de 21 & 0 & 0 & 0 & 0 \\
\hline Total & $\mathbf{3 2}$ & $\mathbf{1 0 0}$ & $\mathbf{4 5}$ & $\mathbf{1 0 0}$ \\
\hline
\end{tabular}

La distancia desde 0 hasta $10 \mathrm{~km}$, resultó ser la más frecuente en la localidad de las Peñas, en tanto que en Soledad, los mayores porcentajes se encontraron hasta los $5 \mathrm{~km}$ de distancia.

El objetivo de la recolección de PFNM en las localidades de las Peñas y Soledad ha sido presentado en la pregunta 12.- Objeto de la cosecha del producto (Tabla 13).

Tabla 14. Objeto de la cosecha de PFNM en las localidades de las Peñas y Soledad.

\begin{tabular}{|l|l|l|l|l|}
\hline \multirow{2}{*}{ Objeto de la cosecha } & \multicolumn{1}{|c|}{ Las Peñas } & & Soledad & \\
\cline { 2 - 5 } & \multicolumn{1}{|c|}{ Cantidad } & $\mathbf{\%}$ & Cantidad & \multicolumn{1}{c|}{$\%$} \\
\hline venta & 32 & 100 & 45 & 100 \\
\hline consumo & 0 & 0 & 0 & 0 \\
\hline venta- consumo & 0 & 0 & 0 & 0 \\
\hline Total & $\mathbf{3 2}$ & $\mathbf{1 0 0}$ & $\mathbf{4 5}$ & $\mathbf{1 0 0}$ \\
\hline
\end{tabular}

En las dos localidades objeto de éste estudio es la utilización del fruto de Bonellia sprucei para la venta. Pero los encuestados de las localidades no están de acuerdo y tampoco es favorable la venta del barbasco, aunque se comprobó en los muestreos que el fruto de este PFNM se aprovecha al máximo. Cabe recalcar que los moradores de las dos localidades consideran que la cantidad de dinero que reciben por una caneca (tacho de 20 litros) del fruto del barbascos es muy bajo ya que en años anteriores el mismo estuvo a mejor precio y siendo el aprovechamiento de este PFNM el sustento de cada familia.

Tabla 15. Resultados de la indagación relacionada con la época de recolección de Bonellia sprucei en las localidades de las Peñas y Soledad

\begin{tabular}{|l|l|l|l|l|}
\hline \multirow{2}{*}{ Opciones } & Las Peñas & \multicolumn{3}{l|}{ Soledad } \\
\cline { 2 - 5 } & Cantidad & $\mathbf{\%}$ & Cantidad & \% \\
\hline temporada lluviosa & 7 & 22 & 10 & 22 \\
\hline temporada seca & 17 & 53 & 26 & 58 \\
\hline todo el año & 8 & 25 & 9 & 20 \\
\hline Total & $\mathbf{3 2}$ & $\mathbf{1 0 0}$ & $\mathbf{4 5}$ & $\mathbf{1 0 0}$ \\
\hline
\end{tabular}

En Las Peñas el 53\% de los encuestados aseguran que su recolección de frutos del árbol de barbasco es en la temporada seca, pero un $22 \%$ afirma que lo hace en temporada lluviosa y el restante lo realiza todo el año. En la localidad de Soledad de igual manera el mayor número de encuestados aseguran que se recolecta en la temporada seca.

Bonellia sprucei como Componente Florístico del Bosque Seco Tropical en las Localidades de Las Peñas y Soledad de la parroquia Julcuy 
En la tabla 15, se presenta la abundancia de Bonellia sprucei, obtenida en los sitios de muestreo de Las Peñas y Soledad

Tabla 16. Abundancia de individuos de Bonellia sprucei inventariados en los sitios de muestreo en las dos localidades respectivamente

\begin{tabular}{lllll}
\hline \multirow{2}{*}{ Transectos } & \multicolumn{2}{l}{ Comunidades } & \\
\cline { 2 - 4 } & \multicolumn{2}{l}{ Las peñas } & Soledad & Abundancia \\
\cline { 2 - 4 } & 1 & 23 & 5 & 28 \\
& 2 & 14 & 6 & 20 \\
& 3 & 9 & 8 & 17 \\
& 4 & 5 & 11 & 16 \\
& 5 & 7 & 9 & 16 \\
& 6 & 8 & 13 & 21 \\
& 7 & 6 & 6 & 12 \\
& 8 & 8 & 10 & 18 \\
& 9 & 6 & 5 & 11 \\
& 10 & 7 & 6 & 13 \\
\hline Total & $\mathbf{1 0}$ & $\mathbf{9 3}$ & $\mathbf{7 9}$ & $\mathbf{1 7 2}$ \\
\hline
\end{tabular}

En la (Tabla 16) se presentan los resultados de la densidad y la abundancia de Bonellia spruceien los sitios de muestreo de Las Peñas y Soledad.

Tabla 17. Densidad y abundancia de la especie Bonellia sprucei en los sitios de muestreo de Las Peñas y Soledad

\begin{tabular}{ll}
\hline Parámetros & Total \\
\hline Densidad indv/ha & 86 \\
Abundancia indv/ha & 2 \\
\hline \multicolumn{2}{c}{ indv/ha: Individuos por ha }
\end{tabular}

\section{Curvas de rango-abundancia}

En la (Figura 2) se muestran las curvas de abundancia relativa obtenidas de las especies más abundantes en las dos localidades involucradas en la investigación. 


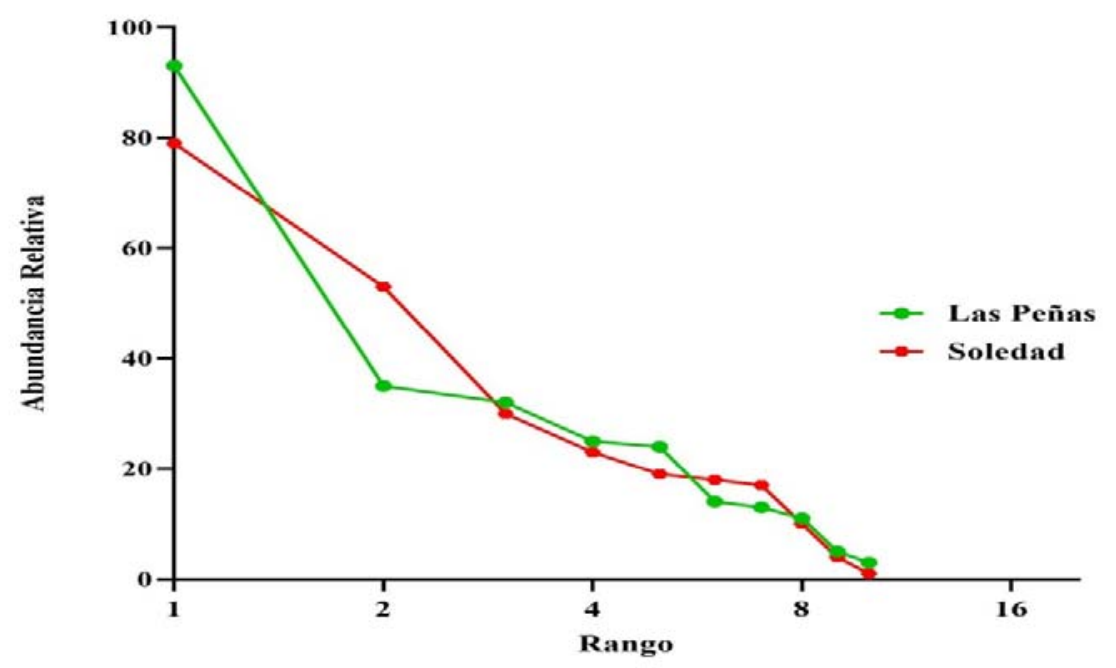

Figura 2.Curvas de Rango-abundancia para las especies más importantes en las localidades de las Peñas y Soledad de la parroquia Julcuy

En relación con la secuencia de las especies, esta difiere en las curvas. Claramente Bonelliasprucei ocupa la primera posición en las dos localidades, en la localidad de Las Peñas la segunda posición la ocupa Prosopissp. y ocupando el tercer lugar la especie de Burseragraveolens, en tanto que en la localidad de Soledad Burseragraveolens ocupa la segunda posición, no obstante, la especie Cordialutease encuentra en la tercera posición dentro de las curvas de abundancia relativa de las especies acompañantes de Bonellia sprucei.

En lo relacionado con el ancho de las curvas difieren muy poco, siendo la Soledad la que muestra una curva más ancha, lo que puede estar indicando que hay mayor equitatividad, demostrado con la distribución de las primeras seis especies en la misma, donde se observa que las pendientes entre una y otra especie, son menos abruptas.

\section{DISCUSIÓN}

Los resultados de la Tabla 1 están en concordancia con la prioridad estratégica número 4, planteada por la FAO (2014b), relacionada con promover el establecimiento y el refuerzo de sistemas (bases de datos) de información sobre los recursos genéticos forestales a fin de abarcar los conocimientos tradicionales y científicos disponibles sobre los usos, la distribución, los hábitats, la biología y la variación genética de las especies y sus poblaciones.

El estudio de los porcentajes y cantidades de usos de los PFNM derivados de Bonellia sprucei en las localidades de las Peñas y Soledad de la parroquia Julcuy, responde a lo publicado por la propia FAO sobre que, existen importantes lagunas en los conocimientos de los recursos genéticos forestales y que la información a nivel nacional se encuentra dispersa y presenta dificultades de acceso, de aquí la pertinencia de esta investigación.

Por otra parte en lo relacionado con la venta y comercialización de PFNM, la FAO (2018) planteó que, se trabaja en la certificación de la producción de PFNM, así mismo con la madera, la 
certificación es solo uno de los indicadores de la sostenibilidad. Los estudios realizados durante los últimos decenios confirman que las medidas "universales" no suelen funcionar: mientras que puede que algunos PFNM estén expuestos a riesgos de sobreexplotación y requieran normas estrictas sobre su extracción, otros productos pueden recolectarse sin poner en peligro la base de recursos, por lo que no necesitan tanta "mano dura".

El hecho de que el 100 por ciento de los encuestados en las comunidades de Las Peñas y Soledad en Julcuy, mencionen que la finalidad del aprovechamiento de Bonellia sprucei sea la venta o la comercialización, está descrito por la FAO (2018), quienes han planteado que, una combinación de enfoques reglamentarios, incentivos económicos y programas de comercialización social (destinados a lograr un cambio de comportamiento) funciona mejor, pues cada factor es necesariopero no suficiente.

Los resultados encontrados en Julcuy acerca de la comercialización de la especie objeto de estudio, coinciden también con los descritos por Arias (2007). Este autor aseguró que, con la forma actual de aprovechamiento, los precios de venta no cubren el costo total de cosecha, transformación y venta del jugo de los frutos de Oenocarpusbataua (Milpeso) en Colombia. Parte del costo está representado en el tiempo de transporte y venta del producto en el mercado; algo similar ocurre con la cosecha y comercialización de Bonellia sprucei.

En lo referente a los resultados obtenidos en la (Tabla 6) relacionada con las partes de las plantas que utilizan los pobladores de las dos localidades estudiadas en Julcuy como PFNM, el total de los puntos porcentuales afirmó utilizar el fruto, lo que hace este aprovechamiento menos agresivo a los árboles. En este sentido Camacho (2008), planteó que, en el aprovechamiento de las especies, los efectos sobre la demografía de plantas dependen de la parte de la planta aprovechada.

El comercio de frutos silvestres muchas veces se dificulta, así FAO (1992), refieren que muchos frutos silvestres comercializados en capitales regionales (...) son desconocidos en Europa y América del Norte y pueden ser susceptibles de exportación.

En Julcuy el mayor aprovechamiento lo realizan del bosque y áreas abiertas, por lo que pueden soportar mayores tasas de aprovechamiento, corroborando lo planteado por (Camacho, 2008), en un estudio de aprovechamiento de hojas de palmas.

A partir de la realización de las encuestas se considera que la distancia para la recolección de PFNM incluye desde 0 hasta $10 \mathrm{~km}$, las viviendas al lugar de recolección, siendo la más frecuente en la localidad de las Peñas, en tanto que, en Soledad, se constató que la distancia se encuentra de $5 \mathrm{~km}$ de distancia en adelante.

La distancia a las actividades humanas (incluye viviendas, instalaciones turísticas, carreteras, caminos, otras relacionadas con el componente antrópico), ha sido descrita por Jiménez et al., (2010); Jiménez (2012); Jiménez et al., (2017), como uno de las variables a tener en cuenta cuando de recursos naturales se trata. 


\section{CONCLUSIONES}

Los PFNM derivados de Bonellia sprucei en las localidades de Las Peñas y Soledad de la parroquia Julcuy pueden incrementarse dadas las potencialidades de usos que esta especie presenta en la región. En el inventario realizado se verificó la asociación de Bonellia sprucei con ocho especies del bosque seco tropical, a saber: Prosopissp, Ceiba trichistandra, Libidibiacorymbosa, Burseragraveolens, Cynophallamollis, Cordialutea, Ziziphusthyrsiflora, y Erythirinavelutina.

\section{REFERENCIAS BIBLIOGRÁFICAS}

Aguirre, M. Z. (2012a). Especies forestales de los bosques secos del Ecuador. Recuperado de https://dspace.unl.edu.ec/jspui/bitstream/123456789/288/1/ZOFRE\%20AGUIRRE\%20MENDOZA.pdf

Aguirre, M. Z. (2012b). Guía para estudiar los PFNM. Documento para estudiantes de la Carrera de Ingeniería Forestal. Loja, Ecuador. Recuperado de https://www.academia.edu/7802645/Guia_para_estudiar_los_productos_forestales_no_maderables_de_Ecu $\underline{\text { ador }}$

Arias, J., C., G., (2007). Oferta de productos forestales maderables y no maderables con potencial económico en un bosque de tierra firme de la Amazonia colombiana. Bosques, fauna y conservación de la Amazonia desde dentro. Recuperado de http://www.scielo.org.co/scielo.php?script=sci_nlinks\&ref=000114\&pid=S0120$0739201400010000500003 \& \operatorname{lng}=$ en

Climate-Data.Org (2019). Clima Julcuy. Recuperado de https://es.climate-data.org/america-delsur/ecuador/provincia-de-manabi/julcuy-179193/

Feinsinger. (2004.). El Diseño de estudios de Campo para la Conservación de la Biodiversidad. Santa Cruz de la Sierra, Bolivia. Editorial FAN.

GraphPad Prism versión 8.0.0 para Windows, GraphPad Software, San Diego, California, EE. UU., Www.graphpad.com". Acceso 19 de julio de 2019.

Hassler, M. (2019). Itis species 2000. Catalogue of Life: 2019 Annual Checklist. Recuperado de http://www.catalogueoflife.org/col/details/species/id/9c7cde4a9ec1eaba7de2ce1f66fb825a

Jiménez, A.; García, M.; Sotolongo, R.; González, M. y Martínez, M. (2010). Productos Forestales no Madereros en la Comunidad Soroa, Sierra del Rosario. Cuba. Revista Forestal Baracoa. , 29(2):83-88. Recuperado de http://www.actaf.co.cu/revistas/rev forestal/Baracoa-20102/FAO2\%202010/PRODUCTOS\%20FORESTALES\%20NO\%20MADEREROS.pdf

Jimenez, A. (2012). Contribución a la ecología del bosque semideciduo mesófilo en el sector oeste de la reserva de la biosfera "sierra del rosario", orientada a su conservación. (Tesis doctorado). Universidad de Pinar del Río, Cuba.

Jiménez, A., Pincay, F.A., Ramos, M.P., Mero, O.F., Cabrera, C.A. (2017). Utilización de productos forestales no madereros por pobladores que conviven en el bosque seco tropical. Revista Cubana de Ciencias Forestales, 5(3), 270-286. Recuperado de http://cfores.upr.edu.cu/index.php/cfores/article/view/264/html

Jiménez, G. A., Macías, E. A. F., Ramos, R. P. M., Tapia, Z. M. V. \&Blandariz, R. S., (2019). Indicadores de sostenibilidad con énfasis en el estado de conservación del bosque seco tropical. Revista Cubana de Ciencias Forestales. Recuperado de http://cfores.upr.edu.cu/index.php/cfores/article/view/409

Molares, S., González, B., Ladio, A. \& Castro, A. (2009). Etnobotánica, anatomía y caracterización físico-química del aceite esencial de Baccharis obovata Hook. et Arn. (Asteraceae: Astereae). Acta Botanica Brasilica, 23(2): 578-589. Recuperado de http://www.scielo.br/pdf/abb/v23n2/v23n2a30.pdf 
Alfredo J. González, Cecibel M.Conforme Quimis, Paola Y. Moreira Aguayo, Lucy M. García Lucas

Morales, V. P. (2012). Estadística aplicada a las Ciencias Sociales Tamaño necesario de la muestra: ¿Cuántos sujetos necesitamos? Madrid. Facultad de Humanidades. Madrid, España: Universidad Pontificia Comillas. Recuperado de http://www.upcomillas.es/personal/peter/investigacion/Tama\%F1oMuestra.pdf

Organización de las Naciones Unidas para la Alimentación y la Agricultura (FAO). (1992). Productos forestales no madereros; posibilidades futuras. Recuperado de http://www.fao.org/3/t0431s/t0431s.pdf

Organización de las Naciones Unidas para la Alimentación y la Agricultura (FAO). (2000). Evaluación de los recursos forestales mundiales 2000 (FRA 2000). Recuperado de http://www.fao.org/3/y1997s/y1997s0g.htm

Organización de las Naciones Unidas para la Alimentación y la Agricultura (FAO). (2014a). Productos Forestales no Madereros. Recuperado de http://www.fao.org/forestry/nwfp/6388/es/

Organización de las Naciones Unidas para la Alimentación y la Agricultura (FAO). (2014b). Plan de acción mundial para la conservación, la utilización sostenible y el desarrollo de los recursos genéticos forestales. Comisión de recursos genéticos para la alimentación y la agricultura. Recuperado de http://www.fao.org/3/ai3849s.pdf.

Organización de las Naciones Unidas para la Alimentación y la Agricultura (FAO). (2018). El estado de los bosques del mundo - Las vías forestales hacia el desarrollo sostenible. (3). Recuperado de http://www.fao.org/3/i9535es/i9535es.pdf

Wong, J. E., Kirsti, T., \& Nell, B. (2001). Evaluaciòn de los Recursos de Productos Forestales No Maderos. Experiencias y Principios Biométricos. Recuperado de http://www.fao.org/3/a-y1457s.pdf 